\title{
Image Inpainting - A Review of the Underlying Different Algorithms and Comparative Study of the Inpainting Techniques
}

\author{
Kaushikkumar R. Patel \\ PG Student, \\ Digital Communication, \\ at NIIST, Bhopal, Madhya \\ Pradesh, India
}

\author{
Lalit Jain \\ Asst.Prof. in Electronics \& \\ Communication Engineering, \\ at NIIST, Bhopal, Madhya \\ Pradesh, India
}

\author{
Ankurkumar G.Patel \\ Asst.Prof. in Computer Sci.\& \\ Engineering at MGITER, \\ Navsari, \\ Gujarat, India
}

\begin{abstract}
Inpainting also known as retouching is the process by which we try to fill in the damaged or missing portions of an image in such a way that it is unable for the person seeing the image to find the fault within the image. Digital Image In painting, a relatively young research area is an art of filling in the missing or corrupted regions in an image using information from the neighboring pixels in a visually plausible manner, while restoring its unity. It is helpfully used for object removal in digital photographs, image reconstruction, text removal, video restoration, special effects in movie discussions and so on. There are numbers of method used for image inpainting. All methods have their own advantage and disadvantages. This paper presents a comparative study and review of different image in painting techniques. The algorithms are analyzed theoretically as well as experimentally.
\end{abstract}

\section{Keywords}

Inpainting, Texture, Structure, Image, Occlusion, Object

Removal, Algorithm, Exemplar

\section{INTRODUCTION}

There are lots of advantages multimedia instruments in today's world peoples are clicking lots of images or picture of theirs and also trying to preserve their past pictures. And as the time passes on, those pictures got damaged (cracks, starches, image data loss, unwanted etc.)

Image In-painting has received considerable attention in the past few years and has become a very active field of research in image processing. The concept of image inpainting commonly known as disocclusion or image completion originates from the medieval art practices, the motive being to recreate the lost or damaged structures in order to bring the medieval art up to date so as to make the damaged artwork more discernible, while conserving its unicity[4],[23].
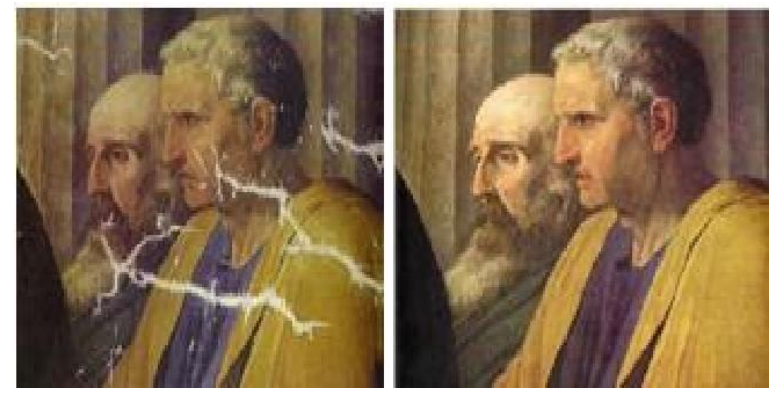

Fig.1: Manual Inpainting performed by a professional artist.
It has been widely used in restoring scratched old photos and old films, text removal from images, digital zooming and special effects in movies, now it is also applied in add or remove objects in photos [13]. Figure 1 shows the manual inpainting done by creative people for centuries to retouch the oil paintings in a room. The inside-painting problem first appeared in the digital domain under the name 'error concealment' in telecommunications where the need was to recreate lost or damaged image structures / blocks that have been lost during data transmission and coding (e.g. streaming video) in order to make it more legible. The principal goal of Digital Image In-painting is to redo the lost or deteriorated components of an image or videos utilizing spatial data from its neighboring countries in order to take the damaged paintings or old pictures close to the original or back to the original state so that it seems natural to the human eye [16]. Many works on Inpainting have been proposed these recent years. The image is to decompose the original image into a structure and a texture image. That reconstruction of each picture is performed individually. The missing structure component is fixed using a structure Inpainting algorithm, while the texture component is repaired by exemplar based synthesis technique [18].

Paper Outline: The next section of this paper presents various digital image inpainting techniques and their experimental results which include PDE based inpainting, Texture synthesis based inpainting, Exemplar based inpainting, Hybrid inpainting and Fast Digital in-painting and Convolution based Method. Results from various techniques are analyzed and compared in Section III. Diverse applications of in-painting techniques talk over in Section IV. At long last, concluding remarks based on experimental results obtained from both real scene photographs and synthetic images are shown in part IV.

\section{INPAINTING ALGORITHMS}

The most challenging task in image in painting technique is the evaluation of the quality of the image so that the inside painted image seems reasonable to the human beholder. The survey contains several inpainting algorithms that were developed.

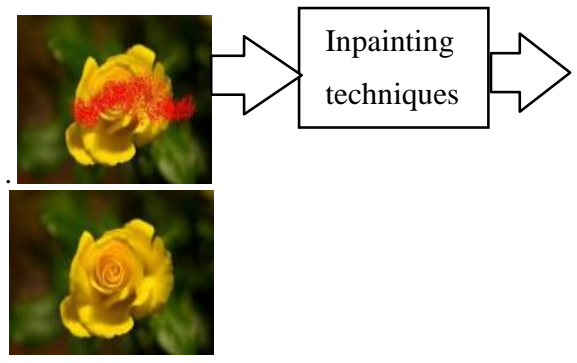

Fig.2: Inpainting method 
Nowadays, there are different techniques of image inpainting are available. Several attacks have been employed by the researchers for digital image in painting which may be separated into the following broad classes:

- $\quad$ Partial Differential Equation (PDE) based In painting.

- Texture Synthesis based In painting.

- Exemplar based in painting.

- Hybrid In painting.

- Fast Digital In painting and Convolution Based Method.

Thus we can easily read the methods and class them into various categories as follows.

\subsection{Partial Differential Equation (PDE) based In painting}

The first digital inpainting technique was the diffusion based in-painting. In this technique the corrupted or the missing part is filled by diffusing information from the surrounding countries into the missing region at the pixel point in a way that the changes seem undetectable to the beholder(observer). Isotropic Diffusion stems from the one-dimensional heat flow equation:

$$
\left\{\begin{array}{l}
I_{t=0}=I_{D} \\
\frac{\partial I}{\partial t}=\Delta \mathrm{I}
\end{array}\right.
$$

Where $I_{D}$ is the deteriorated version of the original image I and $\Delta \mathrm{I}$ is the image Laplacian. The time variable t' denotes the progression of the function $I$ in a sequential continuous manner. Isotropic diffusion, which minimizes the variations acts as a low pass filter that suppresses high frequencies in an image. And it is because of this reason that isotropic method suffers from blur close to the contours. Thus more general methods using nonlinear Partial Differential Equations that were earlier used for fluid dynamics have been introduced to preserve sharpness and edges thereby increasing the efficiency of this method. Figure 4 shows the in-painted output of the damaged image using PDE technique. Bertalmio et.al [20] have proposed a digital image inpainting algorithm based on Partial Differential Equation (PDE) in which the counselling of the lines of equal luminescence i.e. isophotes (figure 5) is maintained by measuring the direction of the largest spatial change obtained by working out a gradient vector and rotating this vector by 90 radians. Bertalmio et.al took the ideas from computational fluid dynamics (CFD) to continue the isophote lines into the region to be in painted [21]. It processes the image intensity as a stream function and the Laplacian of the image as vorticity of the fluid which continues into the area to be in painted by vector fields defined by stream function.

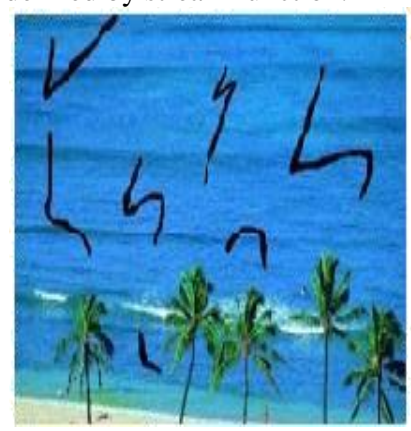

A

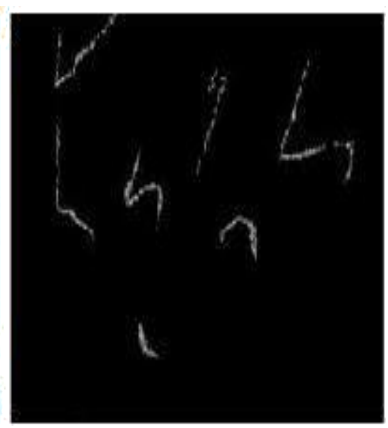

B

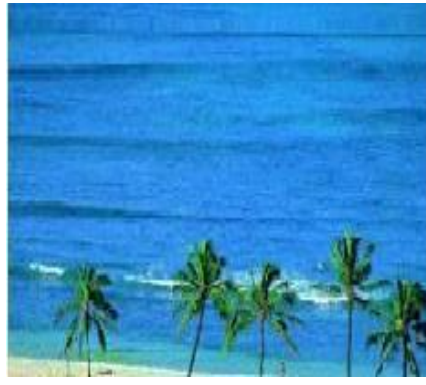

C

Fig.3: In-painted results of PDE based image-painting(a) The Original Image, (b) The mask, (c) The Result

This is done by formulating a partial differential equation which propagates the information (Laplacian of image) in the direction of minimal change using 'isophote lines' (the lines of equal gray value) [8], [9]. This method is thus based directly on the Navier Strokes Equations for CFD. The PDE technique for in painting produces good results if the region to be filled in are small but if the missed regions are large, the efficiency of this algorithm decreases as it takes a long time to fill in large missing areas and the results produced will also be unsatisfactory.

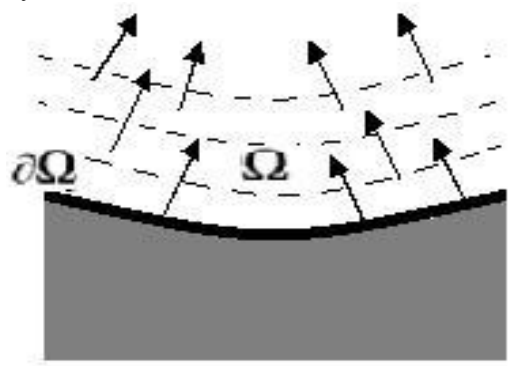

Fig.4: Direction of the melodic phrases of equal gray value (isophot lines).

The first PDE base approach given by Bertalmio [13]. It employs the concept of the isophotes (linear edges of surrounding country) and dissemination process. The primary trouble with this method is that due to blurring effect of diffusion process replication of large texture is not executing good. Pixels on edges are also not covered right. TV (Total Vibrational) model is proposed by Chang and Shen which uses anisotropic diffusion and Euler-Lagrange equation. From TV model, another algorithm presented based on the CDD (Curvature Driven Diffusion model) which include curvature information of the isophotes.

\subsection{Texture synthesis based image inpainting}

PDE based techniques are easily suited for filling in small gaps, text overlays, etc. But PDE technique usually fails if applied to areas containing regular patterns or to a textured field. This failure is because of the following reason:

1. Mostly high intensity gradients are present in grains which may be interpreted wrongly as edges and falsely carried into the region to be painted.

2. In case of PDE based in painting, the information used is simply the boundary condition that is present within a narrow circle around the area to be painted. Therefore, it is unacceptable to recognize structures, textured areas or regular patterns from such an insignificant quantity of data.

In this method, holes are filled by sampling and copying neighboring pixels [19], [21]. The chief dispute between 
different texture based algorithms is how they maintain continuity between hole's pixel and original image pixels. This method is simply running for selected number of images, not with all. Yamauchi et.al presented algorithm which generates texture under different brightness conditions and workplace for multi resolution [3].

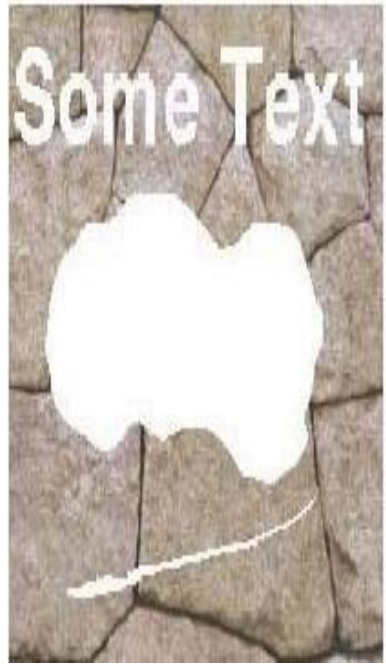

(a) Input Image

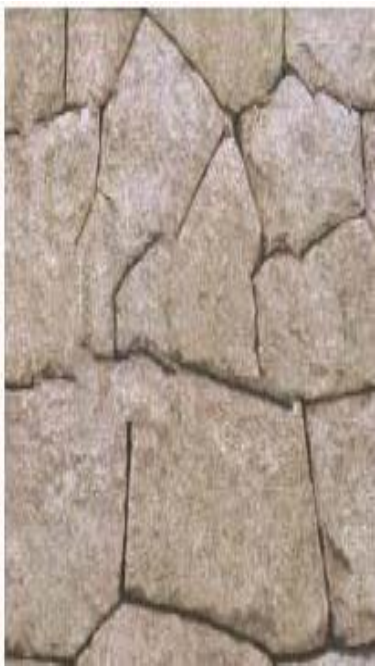

(b) Texture Synthesis
Fig.5: Example of texture synthesis (a) Input Corrupted Image, (b) In-painted output using texture synthesis

The fast synthesizing algorithm presented in [19], uses image quilting (stitching small batches of existing images). All texture based methods are different in terms of their capability to generate texture with different color intensity, gradient and statistical features. Texture synthesis based inpainting method not handle real well for natural images. These methods don't handle edges and boundaries well. In some fonts the user requires to enter which texture to replace with which texture. Hence these methods are used for small area of Inpainting [10]. These algorithms have difficulty in handling natural images. Texture synthesis techniques could be applied in repairing digitized photographs and if the damaged area needs to be filled-in with some regular pattern, texture synthesis does a good job.

\subsection{Exemplar based Inpainting}

The exemplar based approach is an important inpainting algorithm and they have proven to be very effective. Basically it consists of two basic steps: first step priority assignment and the second step is the selection of best matching patch. The exemplar based approach regarding the best matching pieces from the source region, whose similarity is measured by certain metrics, and pastes into the aim field. Exemplar based Inpainting iteratively synthesizes the target neighborhood, by the most similar patch in the source region [8]. According to the filling order, the method fills structures in the missing regions using known information of neighboring regions. Generally, an exemplar-based Inpainting algorithm includes the following main steps:

1) Initializing the Target Region, in which the initial missing areas are extracted and interpreted with appropriate data structures.

2) Computing Filling Priorities, in this a predefined priority function is applied to compute the filling order for all unfilled pixels $p \in \delta \Omega$ in the root of each filling iteration.
3) Searching Example and Compositing, in which the most similar model is explored from the source region $\Phi$ to compose the given patch, $\Psi$ (of size $\mathrm{N} \times \mathrm{N}$ pixels) that centered on the given pixel $\mathrm{p}$.

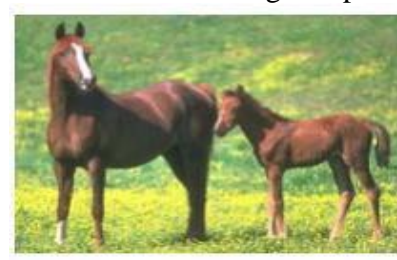

(a)

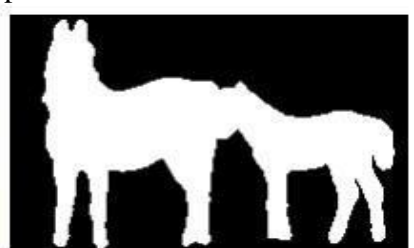

(b)

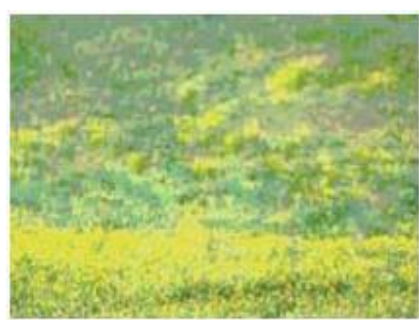

(c)

Fig. 6: Removing Large Objects using Exemplar based Texture Synthesis (a) Original Image, (b) Developed Mask, (c) In-painted Result

4) Updating Image Information, in which the boundary $\delta \Omega$ of the target region $\Omega$ and the required information for computing, filling priorities are updated. Numbers of algorithms are developed for the exemplar based image Inpainting.

Bertalmio [13] produced a hybrid algorithm to combine the diffusion-based system and texture synthesis [14]. This algorithm works well in recovering not only the geometrical structures, but besides the small texture regions. Criminisi [12] developed an effective and uncomplicated access to encourage filling in from the boundary of the missing region where the strength of isophote nearby was strong, and then used the sum of squared difference (SSD) to choose a best matching patch among the candidate source patches. This algorithm of criminisi the region filling order is specified by the priority based mechanism. Cheng [10] generalized the priority function for the family of algorithms given in [12] to provide a more robust operation.

Wong [7] developed a weighted similarity function. That function uses several source patches to reconstruct the target patch instead of utilizing a single source patch J. WU [3] used the structure generation and Bezier curves to make the missing edge information. Using the structural information and reconnecting contours by curve filling process, the damaged regions will be planted. Inwards [13] first decomposing the original image into the entirety of two pictures, one capturing the basic image structure and the other capturing the texture. The first image (structure image) is inpainted following the work of Bertalmio et al. [21], while the other one is filled-in with a texture synthesis algorithm following the work of Efros et al. [22]. The algorithm works well enough for well designed structures in the image, but in the event of natural images the structures do not have well defined boundaries so the effects might not be correct.

In [12] Inpainting techniques fill holes in images by propagating linear structures (called isophotes in the inventing literature) into the target region via diffusion. They are motivated by the partial differential equations of physical heat flow, and work convincingly as restoration algorithms. Their 
drawback is that the diffusion process introduces some blur, which becomes noticeable when filling larger areas. The technique presented here combines the hard spots of both approaches into a single, efficient algorithm. In this story the author proposed an exemplar-based image completion algorithm. Their method computes the priorities of patches to be synthesized through a best-first greedy strategy which depends upon the precedence attributed to each spell on the filling-front, where the patch filling order is defined by the angle between the isophotes direction and the normal way of the local filling front. This algorithm works well in large missing areas and textured areas. The technique is capable of propagating both linear structure and flat texture into the target region with a single, simple algorithm.

In [10] instead of creating a new inpainting scheme, they try to generalize the priority function for the family of algorithms given in [12] to provide more robust performance. In this paper presents, the new priority function is able to resist undesired noises and robust to the aforementioned over amplified phenomenon. The computational complexity of the proposed algorithm is dominated by two tasks: exemplar search and component weight selection. Hence, the limitation of this report will concentrate on the investigation of an efficient searching scheme and on the automatic discovery of component weights for different sorts of pictures.

In [5] they develop the proposed algorithm is basically an extension to the algorithm proposed by Criminisi et al. [12]. Using this algorithm, we can inpaint large missing regions in an image as well as reconstructed small defects. This attack used a priority count based on specifying a means of differentiating between patches that bear the same minimum mean squared error with the selected tour. This technique can be applied to fill minor scratches in the icons/photos as well as to remove larger objects from them. It is also computationally efficient and runs well with larger images.

In [3] a new hybrid image inpainting method based on Bezier curves which combines the exemplar-based inpainting technique and the edge-based image restoration algorithm. For restoring image structures In that first use the segmentation by Otsu's thresholding to obtain the information of edges and Bezier curves used to reconstruct the image skeletons in missing areas. Using this approach we restore approximates incoming edges with only lines and circle arcs which preserves successfully the curvature structures in the damaged picture. These methods solve the disadvantage of Exemplar based approach for handling ambiguities in which the missing region covers the convergence of two countries and achieves better results than the conventional edge based restoration method.

In [2] a robust exemplar-based image in-painting algorithm is proposed. The algorithm uses a novel priority function to regulate the inside-painting order. Structure tensor is adopted in the priority role. Structure tensor contains the transformation direction of the image and the size of transformation in these ways. So, a patch along a strong geometric structure has a higher in-painting priority and would be discharged prior to other pieces to keep the analog structure.

In [1] here they deliver a novel of exemplar-based inpainting as a global energy optimization problem, written in terms of the offset map. The proposed energy function combines a data attachment term that ensures the continuity of reconstruction at the limit of the inpainting domain with a smoothness term that ensures a visually coherent reconstruction inside the butt area. This novel formulation is adjusted to receive a global minimum using the graph cut algorithm. To reduce the computational complexity, they propose an efficient multiscale graph cut algorithm. Propose a multi-scale graph cut algorithm to efficiently resolve the energy minimization problem in which a feature vector representation is brought out to compare patches at low resolution, to redress the information loss. This office can significantly eliminate ambiguities and improve the accuracy of the offset map. The reasons for using a multi-scale graph cut algorithm are twofold: the first one is obvious to reduce the computational cost, the graph labeling at the original image resolution being computationally intensive because of the large number of labels and pixels in the objective country. The second is that the smoothness term, that uses at each scale the 4 nearest neighbors, can capture more spatial information at the lowest resolution layer. This aids toward off getting stuck into bad local minima. For the same cause, besides the multi-scale strategy is used in the Patch Match method.

\subsection{Hybrid Inpainting}

Partial differential equation based methods perform well for low and sparingly distributed gaps. They are suitable for sparse smooth images and for disseminating strong structures, but PDE based methods are unable to synthesize textures [22]. On the other hand, exemplar based in painting performs well in synthesizing textures with regular/homogenous patterns. Unlike PDE they do not maintain the linear structure of images with small holes spread over most of the image field.

Natural images are composed of composite structures and textures. The structures comprise of primary sketches (corners, edges) of an image and textures are regions with regular/ homogenous feature statistics. A technique is therefore needed to handle complex images containing both structures and textures.

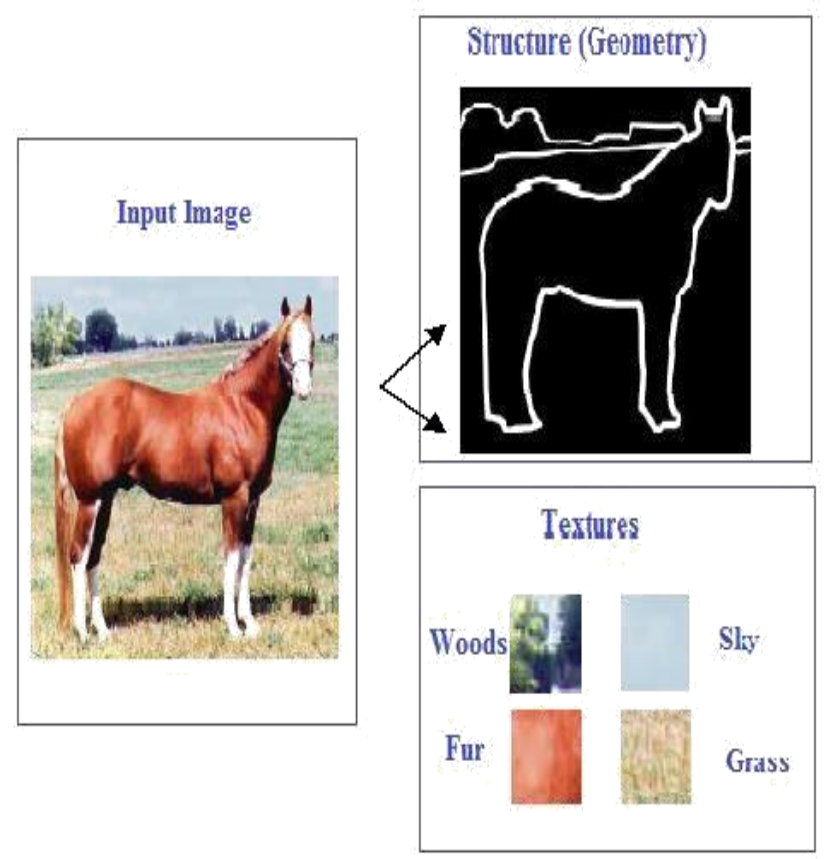

Fig.7: Decomposition of an image into Structural (geometric) and textural components 
Hybrid image in painting technique mostly used for filling large missing regions preserve both the texture and the structure of an image so that the changes in the in painted area are undetectable to the human eye. The hybrid approaches combine both texture synthesis and PDE based Inpainting for completing the objective country. The main idea behind these approaches is that it cracked down the icon into two separate parts, Structure region and texture regions [17].

The decomposed regions are occupied by edge propagating algorithms and texture synthesis techniques. These algorithms are computationally intensive unless the fill area is low. This technique uses a two-step approach: the first stage is structure completion done by texture synthesis. The second step consists of synthesizing a texture and color information in each segment, again using tensor voting [14].

\subsection{Fast Semi-automatic Inpainting and Convolution based Method}

Depending on the size of the gap that needs to be filled in, all the inside painting techniques discussed earlier require minutes to hours for an in painting algorithm to finish to fully in paint the missing areas, thereby making it inefficient for interactive user applications.. Therefore, a novel class of fast in painting techniques is developed to accelerate up the conventional in painting techniques. A Telea introduced a fast marching algorithm almost similar to PDE technique, but considerably simpler and faster to implement than other PDE methods [15]. This algorithm computes smoothness of an image from already known neighborhood of an image as a weighted sum to in paint the gap [19].

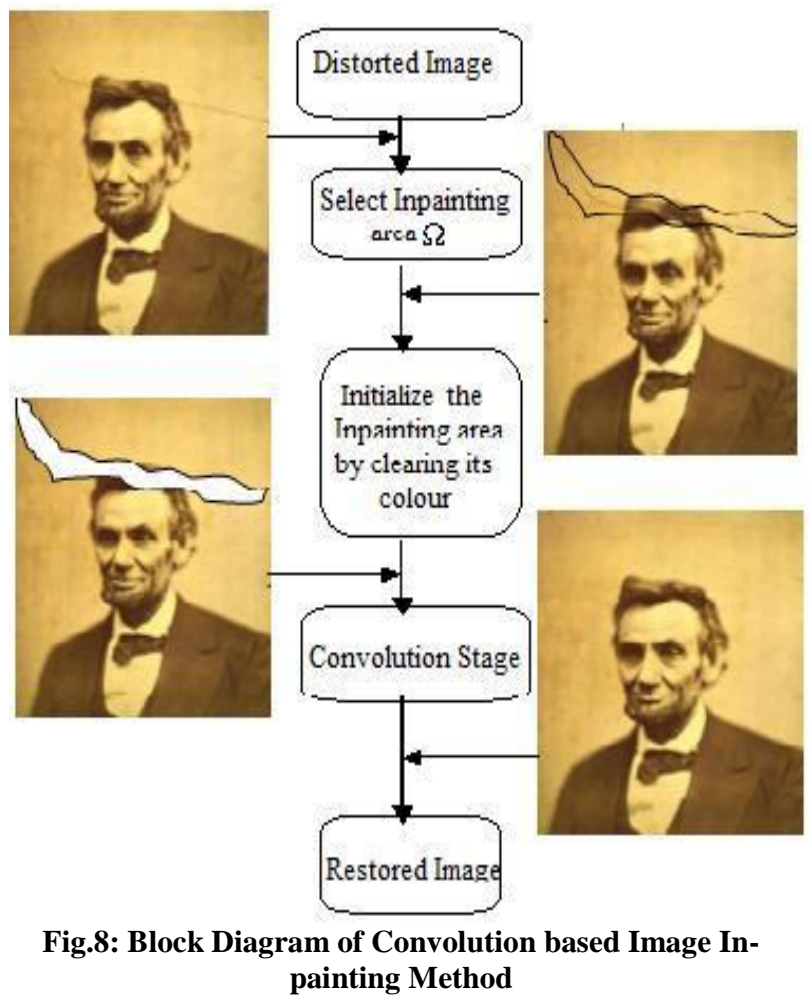

The restriction of this method is it produces blur when the area to be in painted is thicker than ten pixels. Semi-automatic image inpainting requires user assistance in the form of guidelines to assist in structure completion. The method by Jian et.al [6] proposed inpainting with Structure propagation. This technique completes within a two-step operation. In the first step a user manually selects missing information in the hole by sketching object boundaries from the source to the butt area and then a patch based texture synthesis is used to generate the grain. For multiple objects, the optimization is a great deal more difficult and then proposes approximated the answer by using belief propagation. All the methods discussed above will take minutes two hours to fill out depending on the size of the Inpainting area and hence making it unacceptable for interactive user applications. To hurry up the image Inpainting algorithms, new categories of fast Inpainting techniques are being developed. A new method which treats the missing regions as level sets and uses Fast Marching Method (FMM) to propagate image information has been proposed by Thalia in [11]. These fast techniques are not suitable in filling large hole regions as they lack explicit methods to implant edge regions. This technique results in blur effect in the photo.

It is observed that the PDE based image Inpainting algorithms cannot fill the large target (missing) region and it cannot rejuvenate the texture shape. Based on theoretical analysis exemplar based Inpainting will produce sound results for In painting the large missing region also these algorithms can imprint both structure and textured image as well. But they function well only if the missing region consists of simple structure and texture. The Exemplar based technique combines the strong points of both approaches into a single, efficient algorithm. As with inpainting, we pay extra attention to linear structures. But, linear structures abutting the target region only influence the fill order of what is at the core an exemplar based texture synthesis algorithm. The outcome is an algorithm that delivers the efficiency and qualitative performance of exemplar based texture synthesis, but which also observes the image constraints imposed by surrounding linear structure. Here we can briefly survey on Exemplar based Image Inpainting.

\section{RESULTS, ANALYSIS AND DISCUSSION}

Digital image in-painting has become a hotspot and has received a lot of attention since a decade. Various advances have been presented with varying applicability in disocclusion, texture synthesis, object removal, text removed, and image reconstruction. Table 1 shows the merits and the demerits of different in-painting approaches. Established along the varying applicability of different in-painting techniques, all the approaches are as significant in diverse fields of application.

Table 1 Comparison of image Inpainting methods

\begin{tabular}{|c|c|c|}
\hline METHODS & MERITS & DEMERITS \\
\hline $\begin{array}{c}\text { Partial } \\
\text { Differential } \\
\text { Equation (PDE) }\end{array}$ & $\begin{array}{c}\text { Fine Outcome } \\
\text { \& preserves All } \\
\text { Structural } \\
\text { information }\end{array}$ & $\begin{array}{c}\text { Results display } \\
\text { When arring artifacts } \\
\text { Large missing } \\
\text { Regions }\end{array}$ \\
\hline $\begin{array}{c}\text { Total variation } \\
\text { In-painting } \\
\text { (TV) }\end{array}$ & $\begin{array}{c}\text { Works well for } \\
\text { Removing salt } \\
\text { And pepper } \\
\text { Noise }\end{array}$ & $\begin{array}{c}\text { Models Inpainting } \\
\text { Only in small/ } \\
\text { Miniature regions }\end{array}$ \\
\hline
\end{tabular}




\begin{tabular}{|c|c|c|}
\hline $\begin{array}{l}\text { The curvature } \\
\text { Driven Diffusion } \\
\text { Model (CDD) }\end{array}$ & $\begin{array}{c}\text { Used for bigger } \\
\text { Areas }\end{array}$ & $\begin{array}{c}\text { Connects only } \\
\text { Few broken edges }\end{array}$ \\
\hline $\begin{array}{l}\text { Texture Synthesis } \\
\text { Based In-painting }\end{array}$ & $\begin{array}{l}\text { Results do not } \\
\text { Display blurs/ } \\
\text { Artifacts }\end{array}$ & $\begin{array}{l}\text { Not applicable for } \\
\text { Thick scratched } \\
\text { Regions \& curved } \\
\text { structure }\end{array}$ \\
\hline $\begin{array}{l}\text { Exemplar } \\
\text { Based } \\
\text { Texture } \\
\text { Synthesis }\end{array}$ & $\begin{array}{c}\text { Gives } \\
\text { Impressive } \\
\text { Results and } \\
\text { Preserves all } \\
\text { Structural \& } \\
\text { Textural } \\
\text { Information }\end{array}$ & $\begin{array}{c}\text { Gives } \\
\text { Unsatisfactory } \\
\text { Results of the } \\
\text { Corrupted region } \\
\text { Is spread along } \\
\text { Most of the image } \\
\text { Area }\end{array}$ \\
\hline $\begin{array}{l}\text { Hybrid In- } \\
\text { Painting }\end{array}$ & $\begin{array}{c}\text { Restores } \\
\text { Smoothness and } \\
\text { Preserves both } \\
\text { The linear } \\
\text { Structure and } \\
\text { Texture of Image }\end{array}$ & $\begin{array}{l}\text { Produces blocky } \\
\text { Effect } \\
\text { If the damaged } \\
\text { area is large \& the } \\
\text { Patch size is } \\
\text { Inappropriate }\end{array}$ \\
\hline $\begin{array}{c}\text { Convolution } \\
\text { Based In-painting } \\
\text { Method }\end{array}$ & $\begin{array}{l}\text { Produces fine } \\
\text { Results without } \\
\text { Blurring }\end{array}$ & $\begin{array}{l}\text { For corrupted } \\
\text { Region thicker } \\
\text { Than ten pixels, } \\
\text { blurring occurs }\end{array}$ \\
\hline
\end{tabular}

\section{CONCLUSION AND FUTURE WORK}

In this comparative study, we review the existing techniques of image Inpainting. We also have provided a concept and a description of the different Inpainting.

From this analysis, we also highlighted a number of shortcomings and limitations of these different techniques. Here, we highlight some of the possible research areas which can merit future investigations to extend the art of in-painting.

(i) Extension to 3-D: - For the last few years, attempts have been made to extend the in-painting techniques from two dimensions (2-D) to three dimensions (3-D). This modified methodology would help in the restoration of historical artifacts and damaged monuments. This is because the objects that are symmetrical in three dimensions (3-D) may not appear symmetric in their 2-D projection / image plane. However, extensive research is still needed in this direction for the results to be visually pleasing.

\section{(ii) In-painting for high resolution images:-}

Reconstruction/In-painting of high resolution images is still an issue of concern. Dealing with high resolution images, the main issue for the inside-painting techniques is a significant increase in the computation time (up to several hours). Thus to quantify the quality of in-painting techniques, new algorithms can be developed that would be able to implant high-resolution images.

(iii) Video In-painting: -Video In-painting still remains a challenging and a crucial task as the arbitrary camera motion, tracking moving objects in a video, and variations in illumination conditions remain difficult problems to be solved and hence complicate the inside-painting performance. Another issue in video in-painting is to preserve the visual coherency of the in-painted results over time.

(iv) Reducing In-painting time: - More efficient algorithms are required to be developed for reducing the computational cost and In-painting time.

(v) Quality Evaluation: -Accessing the quality of the inpainted images is another open problem as no such quality metrics exists that does not depend upon the reference image. So one has to rely on the human visual comparisons for the quality assessment.

\section{REFERENCES}

[1] Yanking Liu and Vicent Caselles," Exemplar-Based Image Inpainting Using Multiscale Graph Cuts", IEEE Transactions On Image Processing, vol. 22, no. 5, May 2013.

[2] Liu Kui, Tan Jieqing, Su Benyue " Exemplar-based Image Inpainting using Structure Tesnor”, International Conference on Advanced Computer Science and Electronics Information (ICACSEI 2013).

[3] Jiunn-Lin $\mathrm{Wu}$ and Yi-Ying Chou Department of Computer Science and Engineering National Chung Hsing University, Taichung, 402 Taiwan" An Effective Content-Aware Image Inpainting Method", Journal of information science and engineering 28, 755-770 (2012).

[4] Alexandra Ioana Oncu Feier. (2012). Digital Inpainting for Artwork Restoration: Algorithms and Evaluation, Master Thesis Report.

[5] Anupam, Pulkit Goyal, Sapan Diwakar, Information Technology, IIIT Allahabad, India "Fast and Enhanced Algorithm for Exemplar Based Image Inpainting", Fourth Pacific-Rim Symposium on Image and Video Technology 2010.

[6] $\mathrm{Z}$. $\mathrm{Xu}$ and $\mathrm{S}$. Jian, "Image inpainting by patch propagation using patch sparsity", IEEE Transactions on Image Processing, vol. 19, 2010, pp. 1153-1165.

[7] A. Wong and J. Orchard, "A nonlocal-means approach to exemplar-based inpainting", presented at the IEEE Int. Conf. Image Processing, 2008, pp. 2600-2603.

[8] Zhongyu Xu, Xiaoli Lian and Lili Feng. (2008). "Image Inpainting Algorithm Based on Partial Differential Equation", IEEE Computer Society, International Colloquium on Computing, Communication, Control, and Management ISSN:978-0-7695-3290-5.

[9] Michael E Taschler. (2006). "A Comparative Analysis of Image Inpainting Techniques”, The University of York, pp. 01-120.

[10] W. Cheng, C. Hsieh, S. Lin, C. Wang, and J. Wu, "Robust algorithm for exemplar-based image inpainting," in Processing of International Conference on Computer Graphics, Imaging and Visualization, 2005, pp. 64-69.

[11] Telea,"An Image Inpainting Technique Based On The Fast Marching Method", Journal Of Graphics Tools, vol.9, no. 1, ACM Press, 2004.

[12] A Criminisi, P. Pérez, and K. Toyama, "Region filling and object removal by exemplar-based image inpainting," IEEE Trans. Image Process., vol. 13, no. 9 , pp. 1200-1212, Sep. 2004. 
[13] Bertalmio M, Vese L, Sapiro G, Osher S. "Simultaneous structure and texture image inpainting," IEEE Transactions on Image Processing, 2003, 12, 882-889.

[14] Drori D. Cohen-Or, Yeshurun H. "Fragment-based image completion", ACM Transactions on Graphics, 2003, 22, 303-312.

[15] J. Jia and C. K. Tang, "Image is repairing: Robust image synthesis by adaptive $N D$ tensor voting," in Proceedings of IEEE Computer Society Conference on Computer Vision Pattern Recognition, 2003, pp. 643-650.

[16] Shen, J. (June 2003). Inpainting and the Fundamental Problem of Image Processing, SIAM News, 36 (5).

[17] Rane S, Sapiro G, Bertalmio M. "Structure and texture filling of missing image blocks in wireless transmission and compression applications". IEEE. Trans. Image Processing, 2002.

[18] Chan T, Shen J. "Non texture inpainting by curvaturedriven diffusions", Journal of Visual Communication and Image Representation, 2001, 4, 436-449.
[19] Oliviera, B. Bowen, R. Mckenna, and Y. -S. Chang. "Fast Digital Image Inpainting", in Proc. Of Intl. Conf. On Visualization, Imaging And Image Processing (VIIP), pp. 261-266, 2001.

[20] Bertalmio, M., Bertozzi,A.L. And Sapiro, G. (Dec 2001). "Navier Stokes, Fluid Dynamics, and Image and Video Inpainting”, Proceedings of Conf. Comp. Vision, Pattern Rec., Hawai, pp. 355-362.

[21] Bertalmio M, Sapiro G, Caselles V, Ballester C. "Image inpainting", In Proceedings of ACM Conf. Comp. Graphics (SIGGRAPH), 417-424, New Orleans, USA, July 2000.

[22] Alexei A. Efros and Thomas K. Leung, "Texture Synthesis by Non-Parametric Sampling", IEEE International Conference on Computer Vision, pp. 1033 1038, 1999.

[23] Emile-Male, G. The Restorer's Handbook of Easel Painting, Van Nostrand Reinhold, New York. (1976). 